http://dx.doi.org/10.4314/njt.v37i3.38

\title{
IRON REMOVAL IN WASTE WATER USING EXPANDED POLYSTYRENE AS AN ARTIFICIAL MEDIA
}

\author{
J. C. Osuagwu ${ }^{1,}{ }^{*}$, N. L. Nwakwasi ${ }^{2}$ and A. N. Nwachukwu ${ }^{3}$

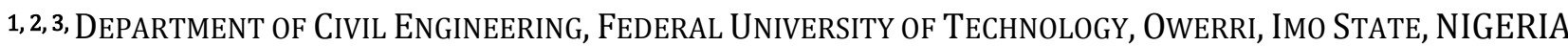 \\ Email addresses: ${ }^{1}$ engrjcosuagwu@yahoo.com, ${ }^{2}$ ngozinwakwasifuto@yahoo.com, \\ 3alphonwabu@yahoo.com
}

\begin{abstract}
This study focused on the use of Expanded Polystyrene as an artificial media for the removal of iron from raw water. The experimental set-up consisted of continuous flow process and the mechanism for iron removal was by adsorption. Water sample of initial concentration $5.5 \mathrm{mg} / \mathrm{l}$ was stored in a 501 container situated at an elevation of $4.4 m$ above the floor and was connected to a plastic transparent container which contained the filter media" Polystyrene". The effects of adsorbent dosage, contact time, $\mathrm{pH}$ and temperature were studied. Results showed that adsorption capacity increased with time for the time range of 0.68-47.6mins investigated and slightly with an increase in temperature until $40^{\circ} \mathrm{C}$ when it began to decrease, suggesting favorable adsorption at medium temperatures. The results showed that the procedure was efficient in reducing iron concentration from $5.5 \mathrm{mg} / \mathrm{l}$ to $3.5 \mathrm{mg} / \mathrm{l}$ within contact time of 5minutes. Better efficiencies could be achieved at higher contact times. The use of EPS material would also offer an avenue for recycling polystyrene widely used in packaging of equipment.
\end{abstract}

Keywords: Contamination, contact time, adsorption, residual iron concentration, pollutants.

\section{INTRODUCTION}

Water is one of the most important necessities for the survival of human beings. Of the total available water on earth, $97 \%$ is sea water and unavailable for human consumption, only $3 \%$ is available as fresh water [1]. Out of this $3 \%$ only a meager $0.06 \%$ can be accessed as the rest comprises of the frozen poles ice cap, groundwater and sewage [2]. The water demand doubles globally every 21 years due to increase in population and industrial activities [3]. The increasing demand of clear water has attracted the attention of government organizations and water industries to develop cost effective technology for water treatment. Raw water treatment is vital to meet the requirements for irrigation, Industry and domestic uses due to increasing population and development in many parts of world.

Iron sometimes referred to as "fly in the ointment" has been one of the most troublesome domestic water contaminants. Various quantities of iron are often present in water because of large amounts of iron present in the soil and because corrosive water will pick up iron from pipes clothing washed in water containing excessive iron may become stained with a brownish colour. Iron deposits may build up in tanks, water heater and pipelines reducing the quantity and quality of water. Iron can often be quite difficult to treat. Iron can be present in several forms, and each form can potentially require a different method of removal. Iron contamination as a result of corroded pipes is a common occurrence in many cities that have very old water systems [5]. Next to hardness, the presence of iron is probably the most common water problem faced by consumers and water treatment professionals. The removal of iron from water requires cost effective techniques that can generate high quality water. Most of the conventional methods of water treatment such as coagulation and flocculation, sedimentation and floatation, membrane filtration, disinfection are either expensive. These Technologies mostly transform pollutants from one phase to another and hardly achieve $100 \%$ elimination. This has led to the need for research and development in the field of iron removal by adsorption.

Adsorption is a process of attracting and retaining molecules on the surface of a solid. The substance that adsorbs on the surface is called adsorbate, and the substance on which it adsorbs is called adsorbent [5]. 


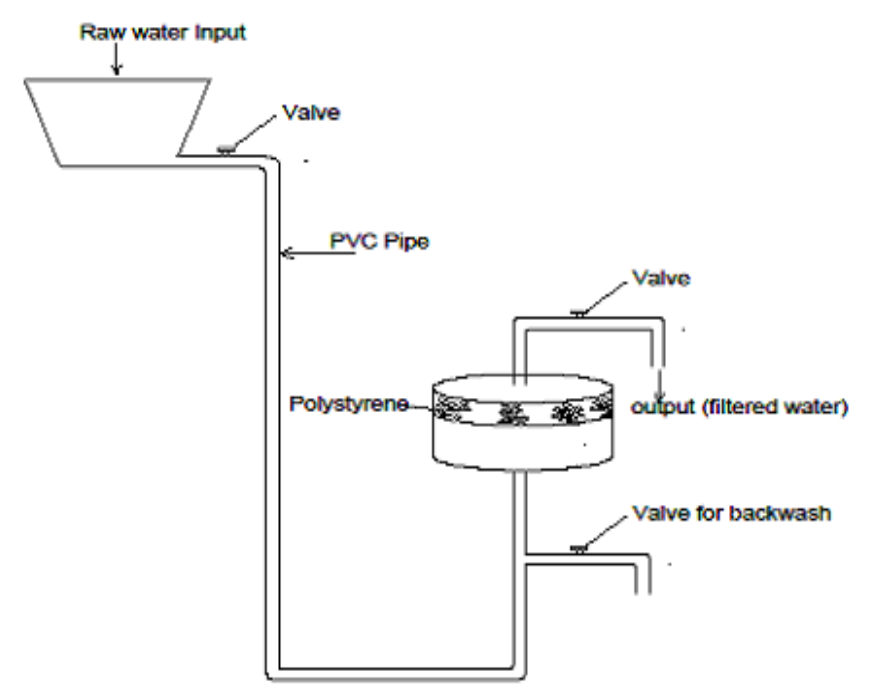

Figure 1: Schematic Representation of the Experimental Layout

Adsorption occurs due to the difference in the properties of the surface of the adsorbent than the bulk. The unbalanced inward force of attraction or free valences at the surface, has the property to attract and retain the molecules onto the surface with which they come in contact [6]. Adsorption is one of the most effective technologies widely used in global environmental protection areas. Adsorption process has not been used extensively in wastewater treatment, but demands for better quality of treated wastewater effluent, including toxicity reduction, have led to an intensive examination and use of the process on activated carbon[7]. It is a process of attracting and retaining molecules on the surface of a solid. The substance that adsorbs on the surface is called adsorbate, and the substance on which it adsorbs is called adsorbent Adsorption occurs due to the difference in the properties of the surface of the adsorbent than the bulk. The unbalanced inward force of attraction or free valences at the surface, has the property to attract and retain the molecules onto the surface with which they come in contact Adsorption process may generate little or no toxic pollutants and involve low initial capital operating costs. It yields treated water with high quality [8]. Modelling of experimental adsorption isotherm data is an essential way for predicting the mechanisms of adsorption science [9].

Polystyrene is a type of polymer with thermoplastic properties produced from the petroleum derived movement, styrene. It is composed exclusively of hydrogen and carbon, the chemical formula $\left(c_{8 H_{8}}\right) n$ It is a lightweight cellular plastic material consisting of small spherical shaped particles containing 98\% air. It is a type of polymer with thermoplastic properties produced from the petroleum derived movement, styrene. It is composed exclusively of hydrogen and carbon, the chemical formula $\left(\mathrm{C}_{8 \mathrm{H}_{8}}\right) \mathrm{n}$ It is a lightweight cellular plastic material consisting of small spherical shaped particles containing $98 \%$ air. The material is environmentally friendly, containing no CFs or HCFCs. It is inert and safe for contact with food products.

This work focused on checking the efficiency of expanded polystyrene (EPS) as a potential filter media for removal of iron from raw water. The specific objectives of study include checking the filtration potentials of polystyrene and its effective application in iron removal by adsorption technique.

\section{MATERIALS AND METHODS}

The experimental procedures involved investigating the effects of four operational parameters on the adsorption of iron using EPS, the parameters include:

(i) Adsorbent dosage

(ii) Flow rate

(iii) Contact time

(iv) $\mathrm{pH}$

(v) Temperature

Water samples for test were collected from borehole at the Federal University of Technology Owerri. The sample was activated by adding iron (II) sulphate, $30 \mathrm{gm}$ of salt was added to $50 \mathrm{~L}$ of water and this increased to the iron concentration from $0.3 \mathrm{mg} / \mathrm{l}$ to $5.50 \mathrm{mg} / \mathrm{l}$. The sample was stored in a $50 \mathrm{l}$ container situated at an elevation of $4.4 \mathrm{~m}$ above the floor and was connected to a plastic transparent container, which contained the artificial filter media "Polystyrene" (Fig 1). The Polystyrene used for the study was sourced from the Polymer and Textile Engineering Laboratory of Federal University Owerri where characterization was done based on Technical data of Polstyrene [10]. The experimental connection was done with a $12 \mathrm{~mm}$ PVC pipe. It consisted of continuous flow process and the mechanism for iron removal was by adsorption. The water sample of known iron concentration was stored in a 50l container situated at an elevation of $4.4 \mathrm{~m}$ above the floor and was connected to a plastic transparent container which contained the filter media" Polystyrene". As a result of the fact that EPS is less dense than water (about $0.02 \mathrm{gm} / \mathrm{cm}^{3}$ ) it floats in water, hence the water sample was made flow against gravity. The filtered water was harnessed through a pipe located above the filtration container and subsequently tested for iron concentration. The mechanism is such the valve next to the water sample reservoir is turned on and water flowing down is 
forced to rise to the filtration container under capillary action. The filtered watered is harnessed through a pipe located above the filtration container. The effect of contact time on the adsorption of iron on EPS was investigated. $40 \mathrm{~g}$ of adsorbent was added to $50 \mathrm{l}$ of water sample of initial concentration $5.5 \mathrm{mg} / \mathrm{L}$.

The flow rate was varied by adjusting the water level in the input container. The fall velocity was determined from Equation 1.

$$
v=a t
$$

where $\mathrm{V}$ is the fall velocity $\mathrm{m} / \mathrm{s}$, a is the acceleration due to gravity $\mathrm{g}\left(\mathrm{m} / \mathrm{s}^{2}\right), \mathrm{t}$ is the fall time in seconds recorded with a stop watch.

$$
Q=A \times v \times
$$

where $A$ is the pipe Cross sectional area

The Runs were carried out for 5 samples for varied flow rates ranging from $0.0035 \mathrm{~L} / \mathrm{S}$ to $0.245 \mathrm{~L} / \mathrm{S}$. The variation between consecutive flow rates was set at $0.06 \mathrm{~L} / \mathrm{S}$ and the variation was kept constant throughout the study of contact time. The resultant filtered water was taken out after each run.

The effect of absorbent dosage was studied by varying the amount of absorbent from $10-50 \mathrm{~g} / \mathrm{l}$. A known amount of absorbent (measured with a sensitive balance) was added to 501 water sample of initial concentrations of $5.5 \mathrm{mg} / \mathrm{l}$.

The effect of $\mathrm{pH}$ on absorption of iron on EPS was also investigated. The $\mathrm{pH}$ of water sample of initial iron concentration of $5.5 \mathrm{mg} / \mathrm{l}$ was adjusted with $0.1 \mathrm{M} \mathrm{HCl}$ and $0.1 \mathrm{M} \mathrm{NaOH}$. A known amount of absorbent was added to the water sample and the $\mathrm{pH}$ value recorded with a pH meter. The iron concentration of the filter of water was determined. The effect of temperature on iron adsorption was investigated by varying the reaction temperature from $25^{\circ} \mathrm{C}$ to $60^{\circ} \mathrm{C}$. Temperatures of raw water used were varied by varied by heating to desired temperatures before the tests were carried out. The mechanism of adsorption was examined by evaluating adsorption data from the experiments. An equilibrium relationship between the amounts of iron adsorbed on the surface of an adsorbent could be established through adsorption isotherms[11]. The analyses were done using Langmuir and Freundlich adsorption isotherms. Due to mathematical simplicity Linear forms of the Isotherm models are widely adopted to determine the isotherm parameters

The Langmuir model is given by the equation

$$
q_{e}=\frac{Q_{o} K_{L} C_{e}}{1+K_{L} C_{e}}
$$

Langmuir adsorption was determined by transforming the equation into linear form.

$$
\frac{1}{q_{e}}=\frac{1}{Q_{o}}+\frac{1}{Q_{o} K_{L} C_{e}}
$$

where: $\mathrm{C}_{\mathrm{e}}$ is the equilibrium concentration of adsorbate $(\mathrm{mg} / \mathrm{L}), \mathrm{q}_{\mathrm{e}}$ is the amount of metal adsorbed per gram of the adsorbent at equilibrium (mg/g), $Q_{o}$ is the maximum monolayer coverage capacity $(\mathrm{mg} / \mathrm{g})$ and $\mathrm{K}_{\mathrm{L}}$ is the Langmuir isotherm constant $(\mathrm{L} / \mathrm{mg})$.

The values of $\mathrm{q}_{\max }$ and $\mathrm{K}_{\mathrm{L}}$ were computed from the slope and intercept of Langmuir plot of $\frac{1}{q_{e}}$ vs $\frac{1}{c_{e}}$. The Freundlich model

$$
q_{e}=K_{f} C_{e}^{\frac{1}{n}}
$$

where $\mathrm{K}_{\mathrm{f}}$ is the Freundlich isotherm constant ( $\left.\mathrm{mg} / \mathrm{g}\right), \mathrm{n}$ is the adsorption intensity, $c_{e}$ is the equilibrium concentration of adsorbate $(\mathrm{mg} / \mathrm{L})$, and $\mathrm{q}_{e}$ is the amount of metal adsorbed per gram of the adsorbate at equilibrium. Linearizing equation, we have

$$
\log q_{e}=\log K_{f}+\frac{1}{n} \log C_{e}
$$

The constant $\mathrm{K}_{\mathrm{f}}$ is an approximate indicator or absorption capacity [12]

\section{RESULTS AND DISCUSSIONS}

\subsection{Effect of Adsorbent Dosage}

The effect of the amount of adsorbent (from $10 \mathrm{~g} / \mathrm{l}$ to $50 \mathrm{~g} / \mathrm{l})$ on iron removal based on initial iron concentration of $5.5 \mathrm{mg} / \mathrm{l}$ is presented in Fig 2. The results show that the residual iron concentration decreased linearly with increase in quantity of EPS in the form of:

$$
y=-0.0384 x+5.3533
$$

where $y$ is the Residual Iron Concentration ( $\mathrm{mg} / \mathrm{l})$ and $\mathrm{x}$ is the mass of EPS (gm)

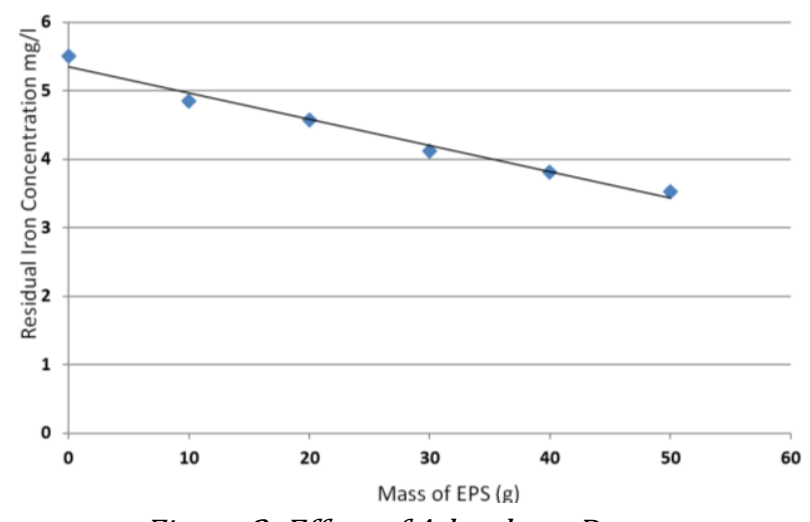

Figure 2: Effect of Adsorbent Dosage

\subsection{Effect of flow Rate}

The effect of rate of flow on the residual iron concentration level is presented in Fig 3. It could be seen that the iron removal efficiency indicated by the residual iron concentration reduced exponentially with 
increase in flow rate. The relationship is presented mathematically in Equation 1.

$$
y=3.7719 e^{1.4568 x}
$$

where $\mathrm{y}$ is the Residual Iron Concentration $(\mathrm{mg} / \mathrm{l})$ and $\mathrm{x}$ is the Mass of EPS (gm)

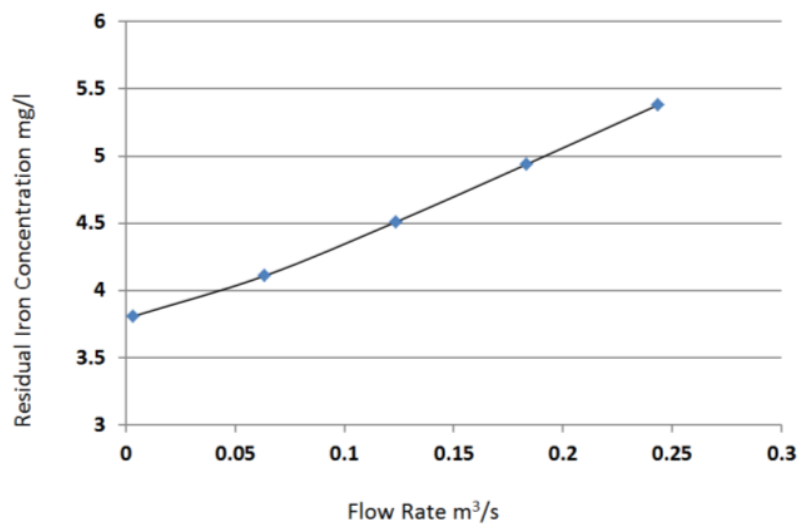

Figure 3: Effect of flow Rate

Results showed a linear increase of efficiency with increase in mass of EPS. It was also observed that rate of flow affected residual iron concentration. Higher flow rates reduced the efficiency of the adsorption process as the residual iron concentration increased with increase in flow rate.

\subsection{Effect of Contact Time}

The reduction of residual iron concentration with increased contact time is illustrated in Fig 3. The variation is defined by Equation 6

$$
y=5.0744 x^{-0.186}
$$

where $y$ is the Residual Iron Concentration (ppm), and $\mathrm{x}$ is the Contact time (mins)

The removal efficiency increased with increase in contact time. The rate was relatively higher at the initial interval. This is attributable to the availability of large number of vacant sites for adsorption of iron.

The relatively high removal of iron initially is attributable to the availability of large number of vacant sites for adsorption of iron.

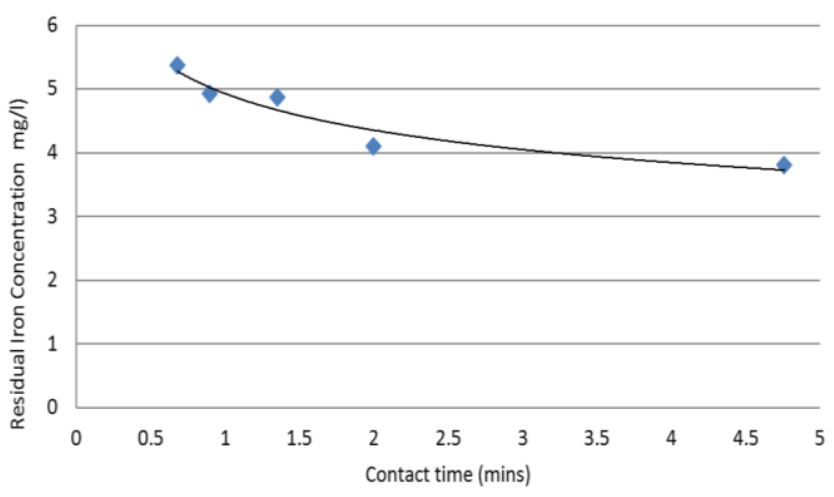

Figure 3: Effect of Contact time

\subsection{Effect of Temperature}

The effect of temperature on the removal efficiency is illustrated in Fig 4. The variation of residual iron concentration with temperature is described by the polynomial equation;

$$
y=0.0011 x^{2}-0.1025 x+5.711
$$

where $\mathrm{y}$ is the Residual Iron Concentration $\mathrm{mg} / \mathrm{l}$, and $\mathrm{x}$ is the temperature ${ }^{\circ} \mathrm{C}$

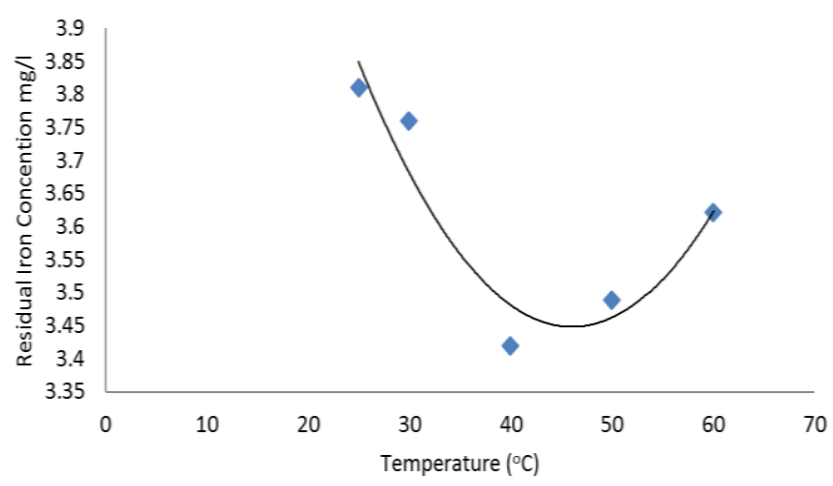

Fig 4 Effect of Temperature

It could be observed that the iron removal efficiency increased with increasing temperature until $45^{\circ} \mathrm{C}$ and beyond this temperature the efficiency decreased. It is possible that the EPS is subjected to chemical degradation beyond this temperature.

\subsection{Effect of $\mathrm{pH}$}

The result is presented in Table 1 . The change in the initial $\mathrm{pH}$ of the raw water sample did not display a significant effect in iron adsorption on the EPS, although adsorption was seen to decrease gradually with increasing $\mathrm{pH}$.

Table 1: Variation of $p H$ with Residual Iron concentration

\begin{tabular}{cc}
\hline $\mathrm{pH}$ & Residual Iron Concentration $\mathrm{mg} / \mathrm{l}$ \\
\hline 7.4 & 3.84 \\
8.0 & 3.84 \\
8.6 & 3.84 \\
9.3 & 3.86 \\
9.9 & 3.88 \\
\hline
\end{tabular}

\subsection{Adsorption Isotherms}

The equilibrium data were examined by two wellknown adsorption isotherm models namely Langmuir and Freundlich models. This confirms that the adsorbate does not form a monolayer on the adsorbent surface but in multilayers. The plots are presented in Fig. 5 and Fig. 6 respectively. The adsorption data exhibited a better fit to Freundlich model. From the 
plot, the value of $\mathrm{q}_{\mathrm{e}}$ is $2.85 \mathrm{mg} / \mathrm{g}$ while $\mathrm{K}_{\mathrm{f}}$ is $5.25 \mathrm{mg} / \mathrm{g}$. This means that $2.85 \mathrm{mg}$ of the iron was absorbed per unit $g$ of Polystyrene.

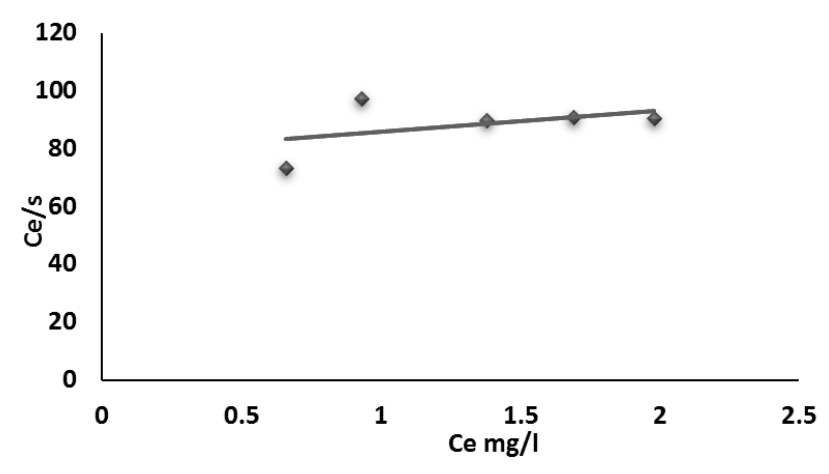

Figure 5: Langmuir Isotherm model for Iron Adsorption

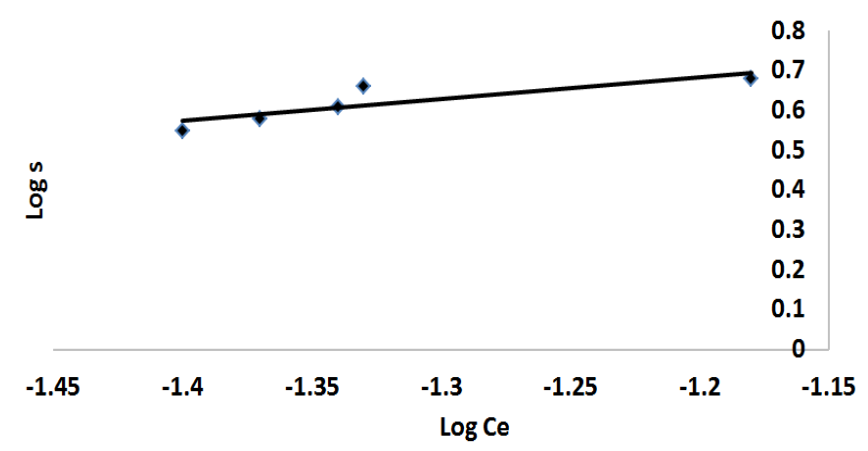

Figure 6: Freundlich Isotherm for Iron Adsorption

\section{CONCLUSION}

The study evaluated expanded Polystyrene for its adsorption capacity. The effect of various operating parameters such as contact time, adsorbent dosage, $\mathrm{pH}$ and temperature were estimated. The results of the study showed the iron adsorption increased with contact time for the small range of time investigated (0.68-47.6mins). The study also showed that iron adsorption increased with the increase in adsorbent dosage. It was also found that iron adsorbent increased gradually till $40^{\circ} \mathrm{C}$ and decreased with an increase in temperature.

The results of the study show that iron removal using floating filter media is adaptable for water quality improvement. Expanded Polystyrene can therefore be a viable adsorbent for the removal of iron from water. Adsorption is safe from the environmental point of view as no sludge is produced. Moreover, the adsorbent can be easily recovered. Iron filtration using Polystyrene can therefore be a cost effective alternative to most widely used conventional methods due to simplicity and flexibility in design coupled with ease of operation. The use of the material would also offer an avenue for recycling polystyrene widely used in packaging of equipment. In Nigeria, the material is a readily available as it is discarded from various homes and offices as packaging material for electronic and household products. Further studies should focus on modifying the properties of expanded polystyrene in order to increase its adsorptive efficiency. Longer contact times should also be adapted to determine the optimum adsorption at various levels of other parameters.

\section{REFERENCES}

[1] Ahuja, S. Handbook of Water Purity and Quality, IWA publishing, London, 2009.

[2] Garbrecht, J. D., Piechhota, T. C. Climate Variations, Climate Change and Water Resourses Engineering. American Society of Civil Engineers. Reston, 2006.

[3] Viessman, W. Jr., Hammer, M. J. Water Supply and Pollution Control, Addison Wesley, Longman Inc. $6^{\text {th }}$ edition. California. USA, 1998.

[4] Asaro, T. Wastewater Reclamation and Reuse. Techonomic Pub.Co. Lancaster, 1998.

[5] Treybal, E. R. Mass Transfer Operations, McGrawHill international editions. Singapore, 1987.

[6] Jiuhiu, Q. U. Research Progress of Novel Adsorption Processes in Water purification: A review. J. Environ. Sci. 20, 1 - 13, 2008.

[7] Metcalf M. and Eddy. Wastewater Engineering, Treatment and Reuse. McGrawhill Ed. Newyork. 1140-1141, 2004.

[8] McKay, G. Use of Adsorbents for the Removal of Pollutants from Wastewaters. CPS Press New York, 1996

[9] Xunjun C. 2015. Modelling of Experimental Adsorption Data: www.mdpi.com/journal/ information; d01.3390/info6010014. Accessed on May 18, 2018. 1996.

[10] Expanded Polystyrene (EPS) Technical Data, Australia: Australian Urethene and Styrene, 2010

[11] Bulut, E., Ozacar, M., and Sengil, I.A. Adsorption of Malachite Green onto Bentonite: Equilibrium and Kinetic Studies and Process Design. Mesopor. Mat., 115, 234-246, 2008.

[12] Vodrias, E., Fytianos, F. and Bozani, E. Sorption Description Isotherms of Dyes from Aqeous Solutions and Waste waters with different Sorbent materials, Global Nest. The Int. J. 4(1) 758, 2002. 